\title{
Glutathione S-Transferase
}

National Cancer Institute

\section{Source}

National Cancer Institute. Glutathione S-Transferase. NCI Thesaurus. Code C16641.

A class of enzymes that catalyze the reaction of glutathione with an acceptor molecule (an arene oxide) to form an S-substituted glutathione; a key step in detoxification of many substances; start of the mercapturic acid pathway. 